\title{
Video Article \\ Transduction of Human Cells with Polymer-complexed Ecotropic Lentivirus for Enhanced Biosafety
}

\author{
Bonnie Barrilleaux ${ }^{1}$, Paul Knoepfler ${ }^{1}$ \\ ${ }^{1}$ Department of Cell Biology and Human Anatomy, School of Medicine, University of California, Davis \\ Correspondence to: Paul Knoepfler at knoepfler@ucdavis.edu \\ URL: https://www.jove.com/video/2822 \\ DOI: doi:10.3791/2822
}

Keywords: Virology, Issue 53, ecotropic, lentivirus, biosafety, oncogenes, cellular reprogramming, induced pluripotent stem cells, cancer

Date Published: $7 / 24 / 2011$

Citation: Barrilleaux, B., Knoepfler, P. Transduction of Human Cells with Polymer-complexed Ecotropic Lentivirus for Enhanced Biosafety. J. Vis. Exp. (53), e2822, doi:10.3791/2822 (2011).

\section{Abstract}

Stem and tumor cell biology studies often require viral transduction of human cells with known or suspected oncogenes, raising major safety issues for laboratory personnel. Pantropic lentiviruses, such as the commonly used VSV-G pseudotype, are a valuable tool for studying gene function because they can transduce many cell types, including non-dividing cells. However, researchers may wish to avoid production and centrifugation of pantropic viruses encoding oncogenes due to higher biosafety level handling requirements and safety issues. Several potent oncogenes, including c-Myc and SV40 large T antigen, are known to enhance production of induced pluripotent stem cells (iPSC). All other known iPSC-inducing genetic changes (OCT4, SOX2, KLF4, NANOG, LIN28, and p53 loss of function) also have links to cancer, making them of relatively high safety concern as well.

While these cancer-related viruses are useful in studying cellular reprogramming and pluripotency, they must be used safely. To address these biosafety issues, we demonstrate a method for transduction of human cells with ecotropic lentivirus, with additional emphasis on reduced cost and convenient handling. We have produced ecotropic lentivirus with sufficiently high titer to transduce greater than $90 \%$ of receptor-expressing human cells exposed to the virus, validating the efficacy of this approach.

Lentivirus is often concentrated by ultracentrifugation; however, this process takes several hours and can produce aerosols infectious to human biomedical researchers. As an alternative, viral particles can be more safely sedimented onto cells by complexation with chondroitin sulfate and polybrene (CS/PB). This technique increases the functional viral titer up to 3-fold in cells stably expressing murine retrovirus receptor, with negligible added time and cost. Transduction of human dermal fibroblasts (HDFs) is maximally enhanced using CS/PB concentrations approximately 4-fold lower than the optimal value previously reported for cancer cell lines, suggesting that polymer concentration should be titrated for the target cell type of interest. We therefore describe the use of methylthiazolyldiphenyl-tetrazolium bromide (MTT) to assay for polymer toxicity in a new cell type. We observe equivalent viability of HDFs after viral transduction using either polymer complexation or the standard dose of polybrene (PB, $6 \mu \mathrm{g} / \mathrm{ml})$, indicating minimal acute toxicity.

In this protocol, we describe the use of ecotropic lentivirus for overexpression of oncogenes in human cells, reducing biosafety risks and increasing the transduction rate. We also demonstrate the use of polymer complexation to enhance transduction while avoiding aerosol-forming centrifugation of viral particles.

\section{Video Link}

The video component of this article can be found at https://www.jove.com/video/2822/

\section{Protocol}

\section{Lentivirus production, harvest, and freezing}

1. Consult your institutional safety official before beginning this protocol, and follow their recommended safety guidelines.

2. Day 1. Start with healthy, rapidly growing 293 T cells to produce virus. Plate the cells at $5 \times 10^{6}$ cells per $10 \mathrm{~cm}$ plate. Use antibiotic-free $293 \mathrm{~T}$ medium (high-glucose DMEM with 10\% FBS and $4 \mathrm{mM}$ L-glutamine) for virus production.

3. Day 2. In the late afternoon, transfect 293T cells as follows (numbers given are for one $10 \mathrm{~cm}$ plate). Let all reagents warm up to room temperature. Pipette $375 \mu \mathrm{l}$ OptiMEM into a microcentrifuge tube, then add $25 \mu \mathrm{l}$ Fugene HD. Do not allow undiluted Fugene to contact the surface of the tube.

4. In a microcentrifuge tube, mix:

- $5 \mu \mathrm{g}$ transfer plasmid (Slc7a1, target vector, or fluorescent control vector)

- $3.75 \mu \mathrm{g}$ packaging plasmid (pCMV-dR8.91 or psPax2)

- $1.25 \mu \mathrm{g}$ envelope plasmid (pMD2.G for pantropic, pHCMV-EcoEnv for ecotropic)

- $\quad$ serum-free OptiMEM to $100 \mu \mathrm{l}$ 
5. Combine the two tubes and incubate the mixture 20-30 minutes at room temperature.

6. Change the medium on the $293 \mathrm{~T}$ cells to $10 \mathrm{ml}$ fresh antibiotic-free $293 \mathrm{~T}$ medium. Add the Fugene/plasmid mix drop-wise to the plate and incubate overnight at $37^{\circ} \mathrm{C}, 5 \% \mathrm{CO}_{2}$.

7. Day 3. Change medium on the 293T cells to $10 \mathrm{ml}$ fresh antibiotic-free $293 \mathrm{~T}$ medium. Be gentle, because $293 \mathrm{~T}$ cells adhere only loosely and can slough off by media pipetted too forcefully onto the monolayer. Incubate for two days in a humidified incubator for virus production.

8. Day 5. Harvest virus and filter with a $0.45 \mathrm{~mm}$ low protein binding filter. Use immediately or dispense into single-use aliquots and freeze at $-80^{\circ} \mathrm{C}$. Frozen pantropic and ecotropic virus should be re-titered after it has been stored for six months and one month, respectively.

9. Titer the virus as follows, using cells that are relatively amenable to transduction. Transduce cells with fluorescent control virus overnight using serial dilutions (e.g. 1:10, 1:100, 1:1000) in fresh medium with $6 \mu \mathrm{g} / \mathrm{ml}$ PB. Change to fresh medium the next day. Allow the cells two days after transduction to begin expressing the fluorescent protein, then determine the fraction of transduced cells by FACS. Calculate the titer in transforming units (TU) per $\mathrm{ml}$, based on dilutions that yield $<15 \%$ transduction to minimize multiple transduction events.

\section{Titer $(\mathrm{TU} / \mathrm{ml})=[(\%$ infected $) \times$ (number of cells in the well) $\times$ (dilution factor) $]$ (volume of virus added to cells)}

\section{Transduction of human target cells with murine retrovirus receptor Slc7a1}

1. Select a multiplicity of infection $(\mathrm{MOI})=2$ to ensure that most cells will be transduced. For target cells that are resistant to transduction such as HDFs, a higher MOI will be required; we dilute the viral supernatant only 1:2 to achieve transduction of a majority of the cells.

2. Transduce target cells overnight with viral supernatant diluted in fresh culture medium with $6 \mu \mathrm{g} / \mathrm{ml} \mathrm{PB}$, which increases transduction by enhancing electrostatic interaction between the virus and target cell. ${ }^{1}$ Ideally one should use a minimal volume of virus, such as $1 \mathrm{ml}$ for a 35 $\mathrm{mm}$ plate, because diffusion is a limiting factor in transduction efficiency.

3. Culture cells for at least 48 hours after removing virus before transducing them with ecotropic virus, in order to ensure sufficient expression of the Slc7a1 receptor.

4. (Optional) It is possible to use blasticidin to select for stably transduced cells expressing Slc7a1, if desired. A blasticidin kill curve should be generated in advance to determine the lowest effective concentration, generally between $2-10 \mu \mathrm{g} / \mathrm{ml}$, that kills all untransduced target cells in 7 days. About 2 days after removing the Slc7a1 virus, switch the transduced cells to medium containing blasticidin. Culture the cells in blasticidin-containing medium for 7 days, at which point all remaining cells will stably express the retrovirus receptor at which point they can be transduced with ecotropic virus as well as banked for future use as a stable Slc7a1-expressing line.

\section{Polymer complex titration to determine toxicity}

1. Day 1. Plate cells at 5000 cells/well in a 96 -well plate, allowing at least triplicate wells for each experimental group. Include untransduced cells in complete medium to provide a baseline value for healthy cells, as well as samples consisting of cells in serum-free medium to induce growth arrest as a control. In parallel, plate cells in an appropriate format (such as a 24-well plate) for microscopic or FACS-based analysis of transduction efficiency in each experimental condition.

2. Day 2. Transduce cells in plates set up for both the MTT and FACS analyses with virus encoding GFP, at an MOI of 0.5 to transduce $\sim 40 \%$ of target cells. Test varied concentrations of CS/PB (e.g. 50, 100, 200, 400, 600 , and $800 \mathrm{mg} / \mathrm{ml}$ of each component), as well as $6 \mu \mathrm{g} / \mathrm{ml}$ PB, to determine optimal amounts. Generate polymer complexes as described in Part 4, below.

3. Day 3. Remove virus-containing medium and replace with fresh medium. Return the plates to a humidified incubator.

4. Day 5. Analyze FACS plate to determine the transduction efficiency with each polymer concentration.

5. Day 6. Remove the medium from all wells of the MTT plate and replace with $100 \mu \mathrm{l} \mathrm{MTT}$ solution ( $1 \mathrm{mg} / \mathrm{ml}$ MTT in complete medium). Culture for three hours in a humidified incubator to allow MTT to be reduced in the mitochondria of metabolically active cells.

6. Remove MTT solution and replace with $200 \mathrm{ml} \mathrm{MTT}$ solvent $(0.1 \mathrm{~N} \mathrm{HCl}, 0.1 \%$ Igepal CA-630 in isopropanol).

7. Incubate for two hours at room temperature or until all of the purple MTT formazan precipitate is dissolved. Read the absorbance on a microplate reader at $570 \mathrm{~nm}$, subtracting the background reading at $690 \mathrm{~nm}$.

8. Select the optimal polymer concentration that produces maximum enhancement of transduction, as determined by FACS, with minimal effect on metabolic activity, as determined by MTT. For HDFs, we selected $100 \mu \mathrm{g} / \mathrm{ml}$ CS/PB based on the data shown in the Representative Results, below.

\section{Ecotropic transduction with polymer complexation}

1. Prepare sterile-filtered stock solutions of $\mathrm{PB}$ and chondroitin sulfate at $20 \mathrm{mg} / \mathrm{ml}$ in water. Aliquot and store at $-20^{\circ} \mathrm{C}$.

2. Pipette viral supernatant into a microcentrifuge tube and dilute to the desired final concentration (e.g. MOI of 2) with fresh culture medium. Add equal volumes of PB and chondroitin sulfate (concentrations determined in step 3 above) in succession, flicking the tube to mix after each addition. The viral mixture will immediately become cloudy as precipitates form. Incubate the viral mixture at room temperature for 5 minutes to allow complex formation.

3. Remove media from the receptor-expressing target cells, replace with the viral mix, and incubate overnight at $37^{\circ} \mathrm{C}, 5 \% \mathrm{CO} 2 . \mathrm{After}$ removing the viral mix, wash the surface of the cells twice with PBS to aid in removing viral complexes. Complete removal is not necessary; we have observed no adverse effects on cell health from residual polymer complexes. 


\section{Verifying specificity of ecotropic transduction}

1. When producing ecotropic virus for the first time, it is prudent from a safety perspective to verify that the virus is unable to transduce unmodified human cells. Transduce human cells with ecotropic lentivirus at relatively high concentration (only a 1 to 2 -fold dilution of viral supernatant in fresh medium) overnight with $6 \mu \mathrm{g} / \mathrm{ml}$ PB or optimized CS/PB concentration.

2. Remove virus-containing medium and replace with fresh medium. After incubating cells for 2 days, validate transgene expression by FACS using fluorescent vectors and/or by RT-PCR with transgene-specific primers.

\section{Representative results:}

Fig. 1A shows the lentivirus production process, and Fig. 1B shows transduction of human cells with ecotropic lentivirus, including pretransduction with murine retrovirus receptor Slc7a1. For titering virus, we use a human rhabdomyosarcoma cell line stably transduced with Slc7a1 (Slc-hRMS). When using fluorescent control vectors to monitor transduction efficiency, we routinely achieve $>90 \%$ transduction efficiency of Slc-hRMS with ecotropic virus, as shown in Fig. 2A and 2B. Transduction rates of HDFs are generally lower because the cells have not been blasticidin-selected for receptor expression (Fig. 2C). Titers of ecotropic lentivirus are generally $10-20 \%$ of VSV-pseudotyped virus when measured on Slc-hRMS (Fig. 2D).

One freeze-thaw cycle reduces titer of ecotropic virus by 16 - 3\%, equal to titer loss during a single freeze-thaw cycle of VSV-pseudotyped virus $(p>0.05)$. Contrary to previous reports, ${ }^{2}$ we observe no positive effect on virus titer from flash freezing in dry ice. Rather, we achieve higher postthaw titers of either pseudotype by simply placing tubes of virus into a $-80^{\circ} \mathrm{C}$ freezer (data not shown).

The MTT viability assay allows sensitive detection of growth arrest in target cells. Transduction with virus plus concentrations of CS/PB up to $800 \mu \mathrm{g} / \mathrm{ml}$ has no effect on HDF metabolism (Fig. 3A). Exposure to CS/PB without virus also has no toxic effect on cells (data not shown). FACS analysis of HDFs transduced with virus plus various concentrations of CS/PB shows enhancement of transduction compared to PB alone (Fig. 3B). The maximum enhancement occurs at $100 \mu \mathrm{g} / \mathrm{ml} \mathrm{CS/PB} \mathrm{(Fig.} \mathrm{3C),} \mathrm{several-fold} \mathrm{lower} \mathrm{than} \mathrm{previously} \mathrm{reported} \mathrm{values.}{ }^{3}$ Thus, it is important to optimize conditions for any given target cell type.

Complexation with CS/PB enhances the observed titer roughly 3-fold in Slc-hRMS (Fig. 4A, $p<0.01$ ). In practice, this yields a greater effect on transduction efficiency at low virus concentrations than at higher concentrations (Fig. 4B), which is most likely due to multiply transduced cells and receptor saturation at higher virus concentrations.

Transduction with ecotropic virus is specific for cells expressing murine retrovirus receptor. When transducing unmodified human cells with ecotropic virus, we have not observed fluorescence greater than the untransduced background whether using PB or CS/PB, as shown in Fig. 5A. Microscopically, HDFs show no transduction in the absence of receptor (Fig. 5B) while pre-transduction with receptor results in fluorescent cells (Fig. 5C).

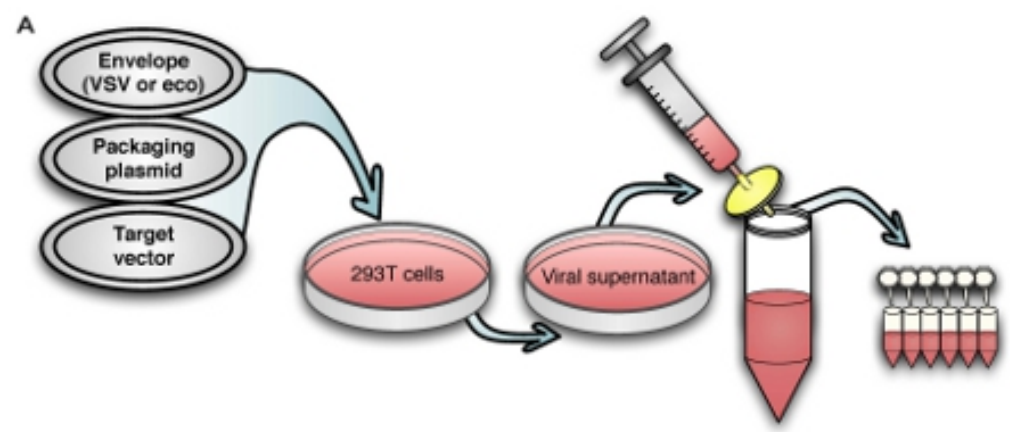

B

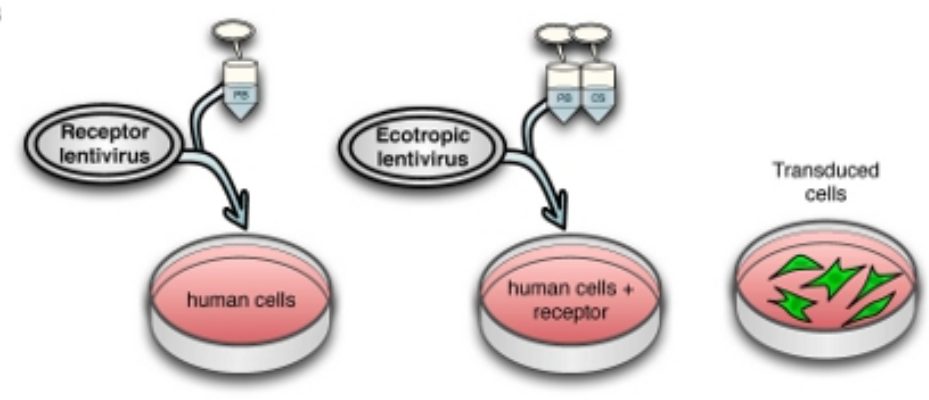

Figure 1. Schematic view of (A) the virus production process and $(B)$ transduction of human cells with murine retrovirus receptor followed by ecotropic lentivirus. 
A)

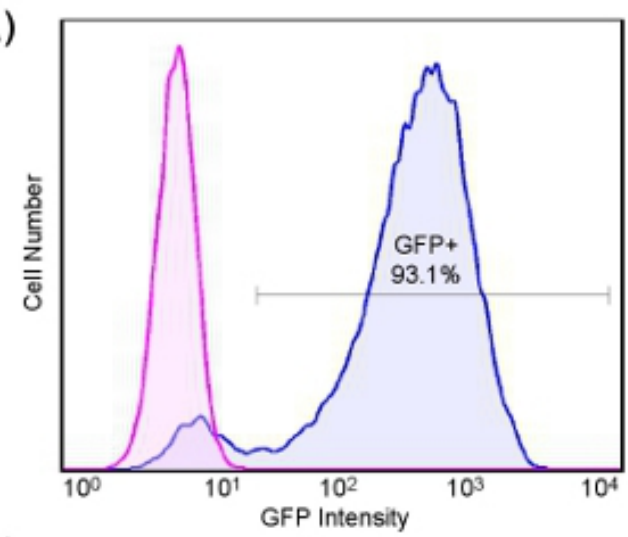

C)

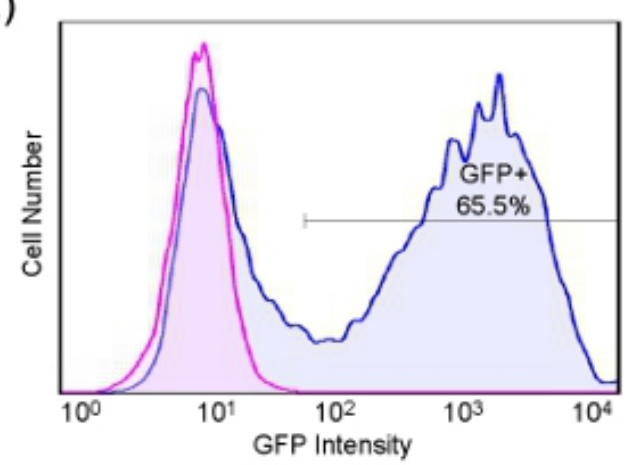

B)

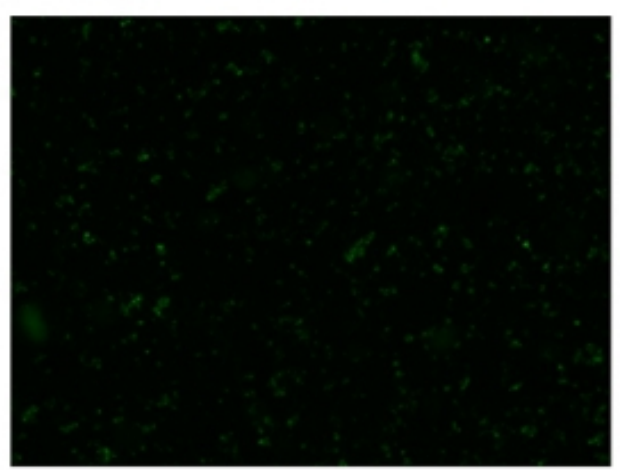

D)

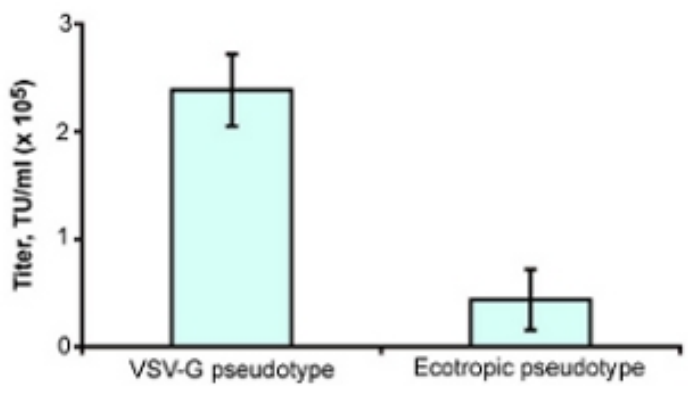

Figure 2. High-efficiency transduction of human cells with ecotropic lentivirus encoding GFP. Cells were pre-transduced with murine retrovirus receptor Slc7a1. (A) Human rhabdomyosarcoma cell line blasticidin-selected for Slc7a1 (Slc-hRMS), transduced (blue) or untransduced (pink) with GFP. (B) Fluorescent micrograph of ecotropic-transduced Slc-hRMS (4X). (C) HDFs transduced (blue) and untransduced (pink) with ecotropic GFP lentivirus; cells were transduced with Slc7a1 two days prior to ecotropic transduction. (D) Titer of VSV-G pseudotyped and ecotropic lentivirus, measured on SIc-hRMS.

A)

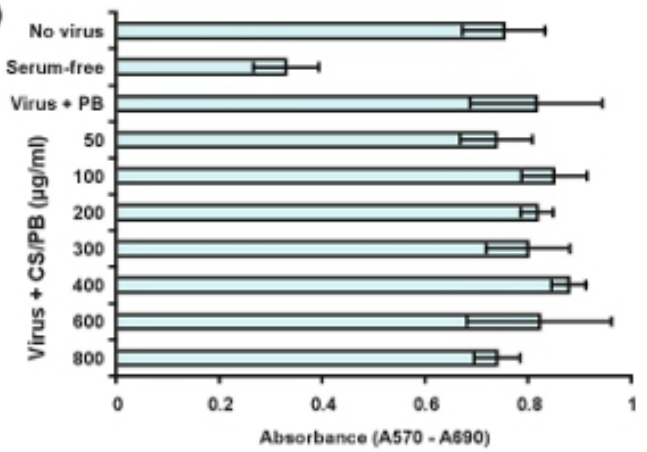

C)

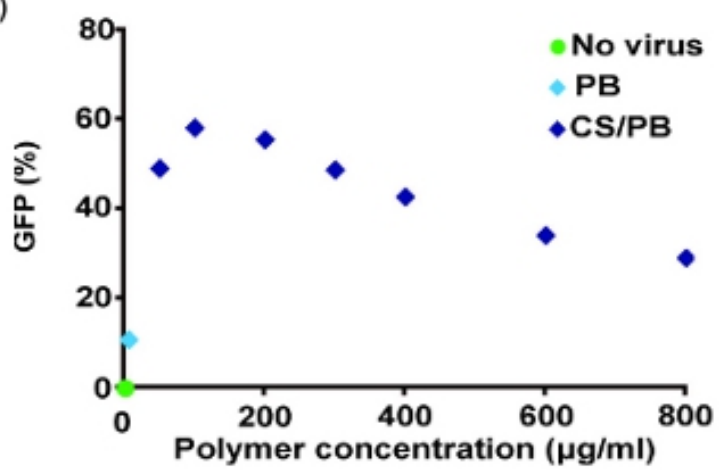

B)

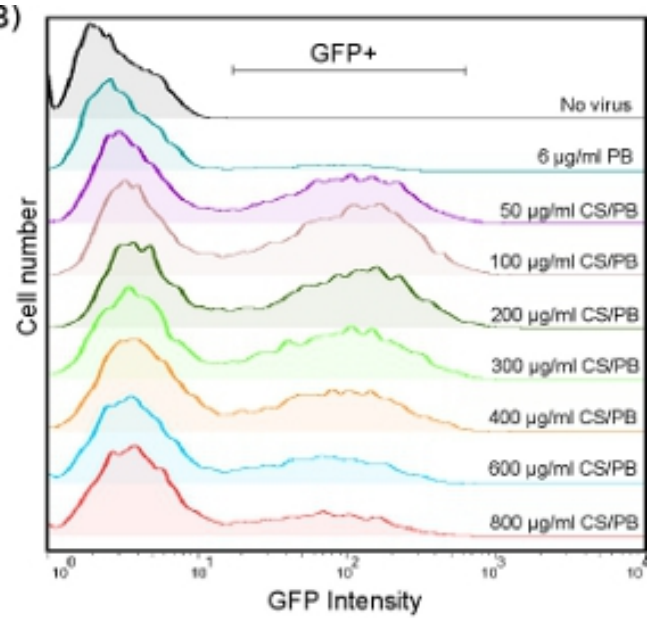


Figure 3. Optimizing chondroitin sulfate/polybrene (CS/PB) concentrations in human dermal fibroblasts. (A) Viability of HDFs measured by MTT assay after transduction in the presence of PB only or with varied concentrations of CS/PB. (B) Enhancement of transduction efficiency in HDFs by different concentrations of CS/PB, measured by FACS. (C) Quantification of transduction enhancement, showing that maximum transduction occurs at $100 \mu \mathrm{g} / \mathrm{ml} \mathrm{CS} / \mathrm{PB}$.
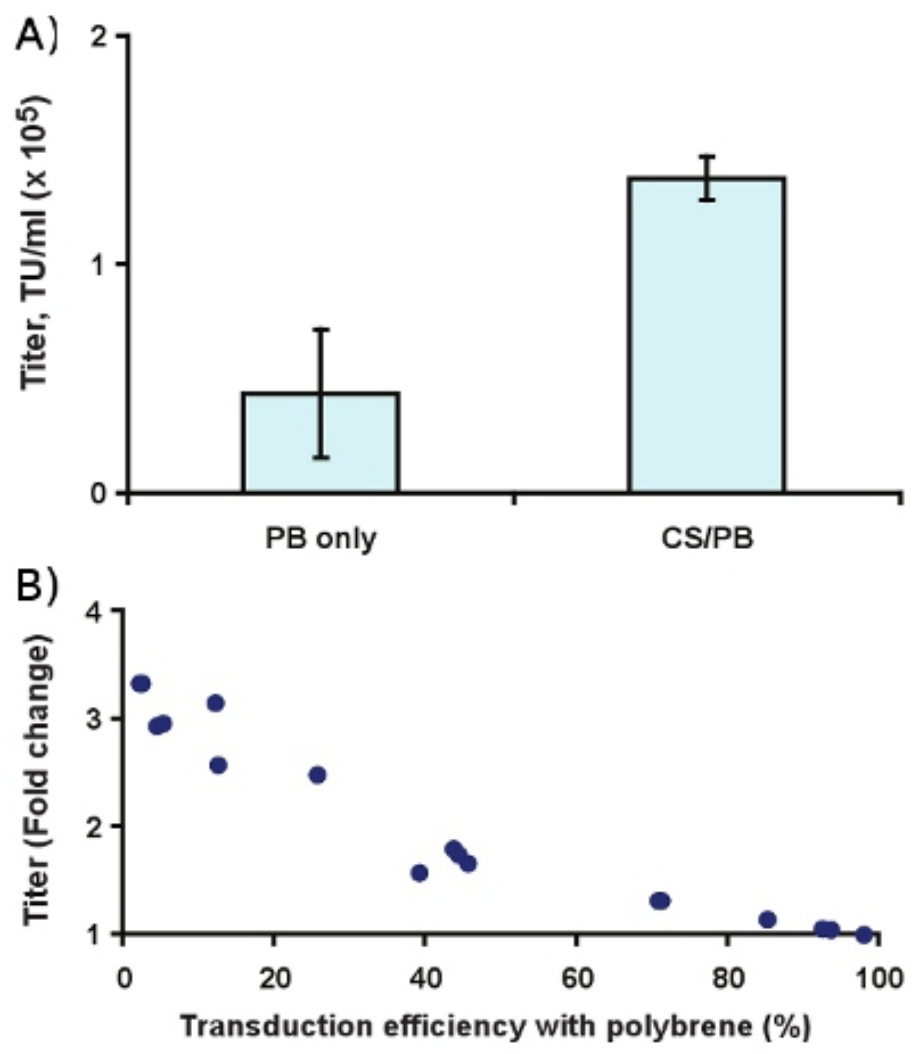

Figure 4. Effect of $400 \mu \mathrm{g} / \mathrm{ml}$ CS/PB on transduction of Slc-hRMS with ecotropic lentivirus. (A) Titer enhancement, and (B) fold change in transduction rate compared to $6 \mu \mathrm{g} / \mathrm{ml}$ polybrene alone.
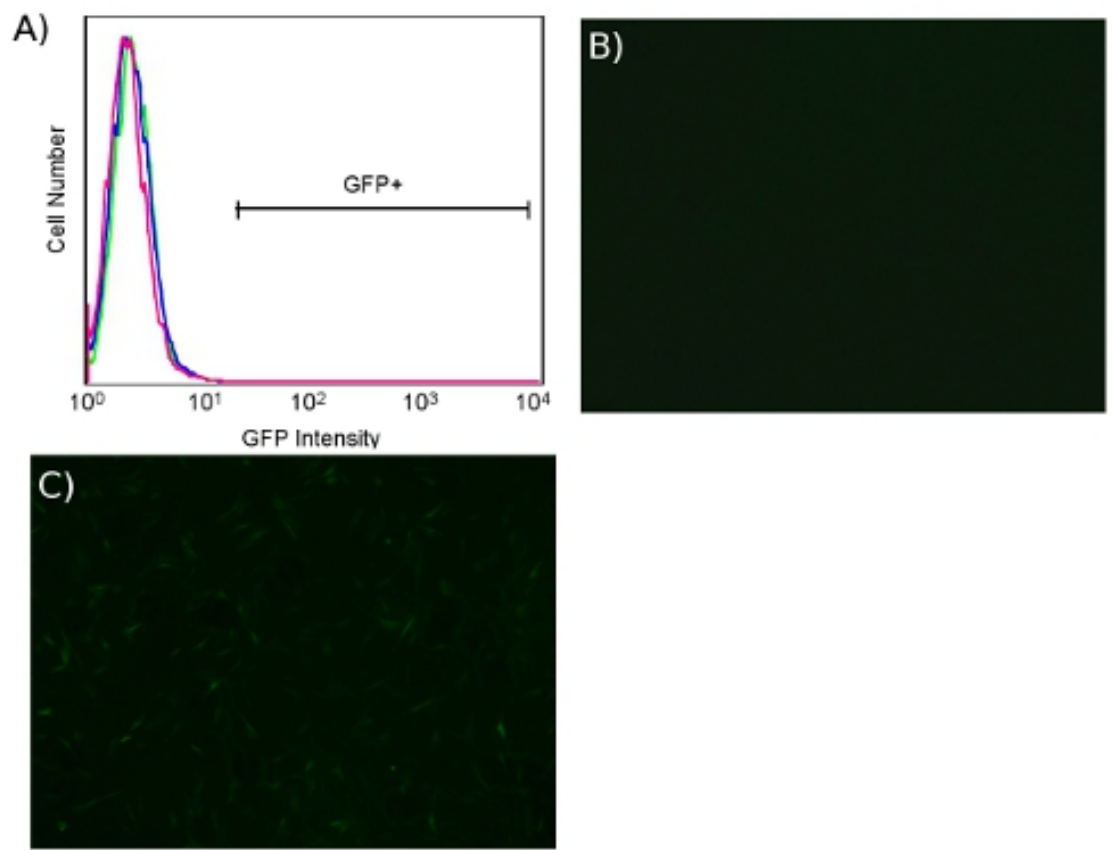
Figure 5. Lack of ecotropic transduction of HDFs in the absence of murine retrovirus receptor Slc7a1. (A) FACS plot of BJ human fibroblasts, untransduced (pink) or transduced (blue) with ecotropic GFP lentivirus in both cases in the absence of receptor Slc7a1. BJ human fibroblasts transduced with ecotropic GFP lentivirus, without (B) and with (C) pre-transduction with receptor Slc7a1 (4X).

\section{Discussion}

Recombinant ecotropic gammaretrovirus based on Moloney murine leukemia virus (MLV) and its receptor mSlc7a1 are well studied and widely available, having been used for over 20 years to deliver transgenes to murine cells. Ecotropic gammaretrovirus has also been used more recently to deliver oncogenes to human cells; in the context of cellular reprogramming, the use of mSlc7a1 to avoid generation of amphotropic virus harboring human oncogenes is well established. ${ }^{4,5}$ However, lentivirus provides significant advantages over gammaretrovirus in transducing refractory cell populations, ${ }^{6}$ including primary cells that are often desirable targets for reprogramming, because the lentiviral pre-integration complex allows transduction of non-dividing cells. ${ }^{7}$

Lentiviruses have been produced with dozens of different pseudotypes, including MLV, in an effort to alter virus tropism, toxicity, and other properties. ${ }^{8}$ MLV-pseudotyped ecotropic lentivirus has been used to transduce mouse cells, ${ }^{9}$ but has seldom been used on human cells. ${ }^{10}$ We therefore propose the use of MLV-pseudotyped ecotropic lentivirus as a safe, cost effective, and highly efficient vehicle to deliver known or suspected oncogenes, including cellular reprogramming factors, to human cells.

It is critical to note that this protocol does not entirely eliminate the need to produce and use pantropic lentivirus; rather, this protocol separates the oncogene(s) from the pantropic virus, insulating researchers from potential self-inoculation with cancer-related viruses. The protein mSlc7a1 and its human homologue hSlc7a1 are ubiquitously expressed amino acid transporters with no known tumorigenicity or ability to confer a selective growth advantage on recipient cells, ${ }^{11}$ making mSlc7a1 of relatively low risk for incorporation into amphotropic virus. This added step may be particularly useful in laboratories lacking dedicated virus or tissue culture facilities of the required biosafety level.

In some situations it may be possible to completely eliminate the use of pantropic virus by transfecting the Slc7a1 plasmid directly into target cells; however, many cells that would be useful targets of this technique are also refractory to transfection. As an alternative, the ability to isolate SIc7a1-transduced cells by blasticidin selection means that VSV-G pseudotyped lentivirus can be used once to generate a stock of receptorexpressing cells, after which ecotropic virus can be used routinely to transduce these cells for many experiments. Researchers should always follow their institutional safety guidelines for working with any lentivirus, regardless of its tropism.

Titers of ecotropic virus achieved with this protocol are moderately lower than VSV-pseudotyped virus, generally 10-20\% of the pantropic virus titer, in agreement with previous studies. ${ }^{9}$ These titers were measured in human cells stably transduced with Slc7a1, so the lower observed titer for ecotropic virus may be partly due to varied expression of the receptor gene in target cells. Nonetheless, the viral titers attained in our protocol are more than sufficient for most applications and lead to transduction of the majority of cells, in some cases $>90 \%$ of cells.

Centrifugation-based techniques are commonly used to concentrate viral particles. However, centrifugation requires several hours, generates infectious aerosols, and can result in significant loss of viral particles. ${ }^{12}$ As an alternative, complexation with CS/PB can be used to enhance transduction without altering viral tropism. ${ }^{3}$ This method is rapid (5 minutes) and inexpensive ( $\$ 0.03$ per 10 ml virus), while roughly tripling the observed titer. No special equipment or proprietary reagents are required, and we observed minimal acute toxicity after exposure of HDFs to CS/ $\mathrm{PB}$, in agreement with previous work in other cell lines. ${ }^{13}$ One potential drawback of this protocol is that some microscopically visible polymer complexes adhere to the cells for several days in culture. We cannot rule out the possibility that these complexes, while not overtly toxic to the cells, may have more subtle effects on cellular processes.

Here we have described a methodology for safely and efficiently transducing human cells with oncogenic factors. This approach should be of great utility to researchers studying oncogenes and stem cell biology including iPS cells.

\section{Disclosures}

No conflicts of interest declared.

\section{Acknowledgements}

Funding for this project was provided by the California Institute for Regenerative Medicine.

\section{References}

1. Davis, H. E., Rosinski, M., Morgan, J. R., \& Yarmush, M. L. Charged polymers modulate retrovirus transduction via membrane charge neutralization and virus aggregation. Biophys J. 86, 1234-1242 (2004).

2. Marino, M. P., Luce, M. J., \& Reiser, J. Small- to large-scale production of lentivirus vectors. Methods Mol Biol. 229, 43-55 (2003).

3. Landazuri, N., \& Le Doux, J. M. Complexation of retroviruses with charged polymers enhances gene transfer by increasing the rate that viruses are delivered to cells. J Gene Med. 6, 1304-1319 (2004).

4. Ohnuki, M., Takahashi, K., \& Yamanaka, S. Generation and characterization of human induced pluripotent stem cells. Curr Protoc Stem Cell Biol. 4, 4A.2 (2009).

5. Hotta, A. et al. EOS lentiviral vector selection system for human induced pluripotent stem cells. Nat Protoc. 4, 1828-1844 (2009).

6. Reiser, J. et al. Transduction of nondividing cells using pseudotyped defective high-titer HIV type 1 particles. Proc Natl Acad Sci U S A 93, 15266-15271 (1996).

7. Cullen, B. R. Journey to the center of the cell. Cell. 105, 697-700 (2001). 
8. Cronin, J., Zhang, X. Y., \& Reiser, J. Altering the tropism of lentiviral vectors through pseudotyping. Curr Gene Ther. 5, 387-398 (2005).

9. Schambach, A. et al. Lentiviral vectors pseudotyped with murine ecotropic envelope: increased biosafety and convenience in preclinical research. Exp Hematol. 34, 588-592 (2006).

10. Koch, P., Siemen, H., Biegler, A., Itskovitz-Eldor, J. \& Brustle, O. Transduction of human embryonic stem cells by ecotropic retroviral vectors. Nucleic Acids Res. 34, e120 (2006).

11. Yoshimoto, T., Yoshimoto, E., \& Meruelo, D. Molecular cloning and characterization of a novel human gene homologous to the murine ecotropic retroviral receptor. Virology. 185, 10-17 (1991).

12. Cepko, C. Large-scale preparation and concentration of retrovirus stocks. Curr Protoc Mol Biol. Chapter 9, Unit 9.12 (2001).

13. Le Doux, J. M., Landazuri, N., Yarmush, M. L., \& Morgan, J. R. Complexation of retrovirus with cationic and anionic polymers increases the efficiency of gene transfer. Hum Gene Ther. 12, 1611-1621 (2001).

14. Zufferey, R. et al. Self-inactivating lentivirus vector for safe and efficient in vivo gene delivery. J Virol. 72, 9873-9880 (1998). 